\title{
SATMF Suppresses the Premature Senescence Phenotype of the ATM Loss-of-Function Mutant and Improves Its Fertility in Arabidopsis
}

\author{
Yi Zhang ${ }^{1}$, Hou-Ling Wang ${ }^{1} \oplus$, Yuhan Gao ${ }^{2}$, Hongwei Guo ${ }^{1,3, *}$ and Zhonghai Li $^{1, *}$ \\ 1 Beijing Advanced Innovation Center for Tree Breeding by Molecular Design, Beijing Forestry University, \\ Beijing 100083, China; yizhang@bjfu.edu.cn (Y.Z.); whling@bjfu.edu.cn (H.-L.W.) \\ 2 Key Laboratory of Pest Management in Crops of the Ministry of Agriculture, Institute of Plant Protection, \\ Chinese Academy of Agricultural Sciences, Beijing 100193, China; gaoyuhanamy@gmail.com \\ 3 Key Laboratory of Molecular Design for Plant Cell Factory of Guangdong Higher Education Institutes, \\ Department of Biology, Southern University of Science and Technology (SUSTech), Shenzhen 518055, China \\ * Correspondence: guohw@sustech.edu.cn (H.G.); lizhonghai@bjfu.edu.cn (Z.L.)
}

Received: 3 October 2020; Accepted: 23 October 2020; Published: 30 October 2020

\begin{abstract}
Leaf senescence is the final stage of leaf development. It is accompanied by the remobilization of nutrients from senescent leaves to developing organs. The occurrence of senescence is the consequence of integrating intrinsic and environmental signals. DNA damage triggered by stresses has been regarded as one of the reasons for senescence. To prevent DNA damage, cells have evolved elaborate DNA repair machinery. The ataxia telangiectasia mutated (ATM) functions as the chief transducer of the double-strand breaks (DSBs) signal. Our previous study suggests that ATM functions in lifespan regulation in Arabidopsis. However, ATM regulatory mechanism on plant longevity remains unclear. Here, we performed chemical mutagenesis to identify the components involved in ATM-mediated longevity and obtained three dominant mutants satmf1 3, suppressor of atm in fertility, displaying delayed senescence and restored fertility in comparison with atm mutant. Molecular cloning and functional analysis of SATMF (suppressor of atm in fertility) will help to understand the underlying regulatory mechanism of ATM in plants, and shed light on developing new treatments for the disease Ataxia-telangiectasia.
\end{abstract}

Keywords: leaf senescence; DNA damage; ATM; fertility; SATMF

\section{Introduction}

Leaves are the primary photosynthetic organs that use the photosynthetic system to fix $\mathrm{CO}_{2}$ to produce carbohydrates, thus providing energy for plant growth and development [1]. Leaf senescence is an inevitable physiological process, accompanied by the nutrient remobilization from senescent leaves to young leaves or other developmental organs [2-4]. Although leaf senescence is a genetically controlled process, it can be induced by various endogenous and environmental stresses [4-10].

Cells have evolved elaborate DNA repair machinery to prevent DNA damage. A growing body of evidence indicates that the loss of DNA damage repair induces premature senescence in animals and plants $[11,12]$. Paradoxically, DNA repair can itself be subject to age-related changes and deterioration [13]. DNA damage repair efficiency also changes during aging, which influences mismatch repair (MMR), base excision repair (BER), nucleotide excision repair (NER), and double-strand break (DSB) repair. Most of the evidence supporting DNA damage-induced senescence comes from the fact that mutations in genes involved in DNA repair lead to multiple premature aging symptoms, supporting the idea that the balance between DNA damage and repair determines the rate of aging [13]. For example, WRN protein, mutated in Werner syndrome (WS) characterized by premature aging in 
young adults, is involved in both homologous recombination (HR) and non-homologous end joining (NHEJ) pathways [14]. Mutation in Lamin A causes Hutchinson Gilford progeria syndrome (HGPS) and also impairs HR [15]. Loss-of-function of the ATM (ataxia telangiectasia mutated) shortens lifespan due to cancer and ischemic heart disease [16,17], and ATM has been reported to be a potential target for alleviating senescence through regulating lysosomal acidification [18]. In eukaryotes, the initial stages of the response to DNA damage are governed by two closely related protein kinases, ATM and ATR (ATM- and RAD3-related) [16]. ATM is activated by auto/transphosphorylation in response to DSBs and leads to the activation of cell cycle checkpoints, DNA repair or apoptosis, while ATR is generally activated by persisting single-stranded DNA breaks (SSBs) $[16,19]$.

Like other eukaryotes, plants have the orthologs of ATM/ATR [20,21]. Functional analyses of ATM or ATR by using knockout mutants reveal that they display overlapping and distinct roles in regulating plant development and in response to DNA damage. Compared with atr mutants, the atm mutants in Arabidopsis are supersensitive to DSB-inducing agents such as methyl methane sulphonate (MMS) or $\gamma$-irradiation [22]. Reverse genetic screening revealed that loss of ATM function by T-DNA insertion or application of a specific kinase inhibitor promotes DSBs-induced and age-dependent leaf senescence, suggesting that ATM is functionally conserved in animals and plants [12]. Moreover, DSBs-regulated genes were overlapped with age-regulated genes, indicating that DSBs induce similar changes on transcript profile with the natural senescence process [12], further supporting the regulatory role of DSBs in aging [11,23].

Our previous work revealed that DSBs increase and DNA repair efficiency decreases as a leaf is aging, and DSBs alone could induce premature senescence, demonstrating the correlation between DSBs and leaf senescence. The PI3K-like protein kinase ATM is involved in repairing age-dependent DNA damage, and the deficiency in ATM resulted in early senescence [12]. Nevertheless, how ATM regulates leaf senescence is still unclear. In this study, we generated transgenic plants overexpressing ATM, which suppressed the early senescence phenotypes of atm mutant. To identify the components involved in ATM-mediated longevity, we performed chemical mutagenesis by treating atm seeds with ethyl methanesulfonate (EMS) [24], and obtained three suppressor lines (samtf1 3, suppressor of atm in fertility), which displayed delayed senescence phenotypes upon treatment with bleomycin (BLM), an inducer of DSBs. Molecular cloning and functional analysis of SATMF (suppressor of atm in fertility) will contribute to understanding the underlying mechanism of ATM in leaf senescence.

\section{Results and Discussion}

\subsection{Overexpression of ATM Delays Leaf Senescence}

Our previous study showed that ATM expression levels decrease as leaf aging and loss-of-function of ATM accelerates leaf senescence process [12]. To further assess whether the expression of ATM mRNA is due to transcriptional regulation, we generated transgenic Arabidopsis plants expressing a GUS ( $\beta$-glucuronidase) gene driven by the ATM promoter containing a $3 \mathrm{~kb}$ fragment upstream of the start codon (pATM:GUS/Col-0). GUS staining of 8-, 16- and 48-d-old pATM:GUS/Col-0 plants showed higher GUS staining in young leaves than in mature or old leaves (Supplementary Figure S1), indicating that ATM transcription declines during plant leaf aging.

We next tested whether constitutive overexpression of ATM could delay leaf senescence. Towards this end, we generated a transgenic line overexpressing ATM under the control of the $35 \mathrm{~S}$ promoter in Col- 0 and atm backgrounds, respectively. Interestingly, we found that overexpression of ATM in wild-type Col-0 background (35S:ATM/Col-0) caused gene co-suppression ( 75\%), leading to atm-like senescence phenotypes, which is not observed in 35S:ATM/atm plants. We examined the senescence phenotypes of the atm and 35S:ATM/atm at different developmental stages. Overexpression of ATM restored multiple abnormal phenotypes of atm mutants, such as small leaves (Figure 1A), slightly early flowering (Figure 1B), and premature leaf senescence (Figure 1C). Compared with Col-0, rosette leaves of the 40-d-old atm mutant exhibited severe yellowing, whereas 35S:ATM/atm leaves 
remained green, suggesting further that ATM is a negative regulator of leaf senescence [12]. We next performed a detailed examination of senescence phenotypes in the third and fourth leaves of wild-type Col-0, atm and 35S:ATM/atm plants. Photochemical efficiency of photosystem II (PSII, Fv/Fm) and chlorophyll contents in atm leaves were significantly less than that in Col-0 and 35S:ATM/atm leaves (Figure 1D,E). In contrast, expression of senescence-associated gene 12 (SAG12), a senescence marker gene, in the atm mutant was more dramatically induced in comparison with Col-0 and 35S:ATM/atm (Figure 1F). Together, these results suggest that overexpression of ATM delays leaf senescence.
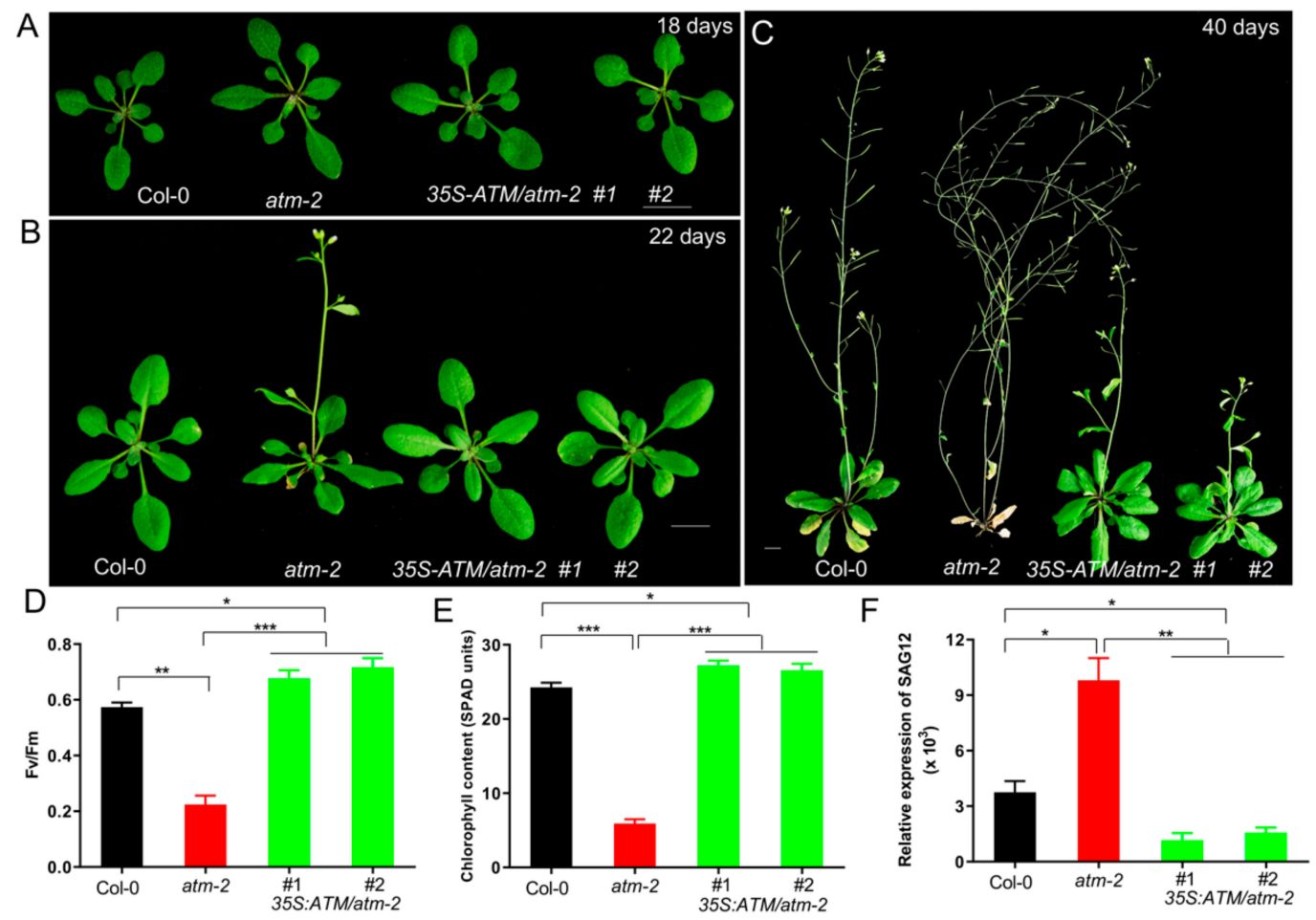

Figure 1. Overexpression of ATM (ataxia telangiectasia mutated) suppresses the early senescence phenotypes of atm mutant. (A) Rosette leaves of 18-day-old plants of Col-0, atm-2 and 35S-ATM/atm-2. (B) Overexpression of ATM suppresses the early flowering phenotype of atm-2 mutant under long-day conditions (18 L/6 D). (C) Senescence phenotypes of 40-day-old plants of Col-0, atm-2 and 35S-ATM/atm-2. (D-F) Photochemical efficiency of PSII (Fv/Fm) (D), chlorophyll contents (E), and qRT-PCR analysis of SAG12 expression (F) in the third and fourth leaves of 35-day-old plants of Col-0, atm-2 and 35S-ATM/atm-2. Three biological replicates were performed. Error bars represent standard deviation (SD, $n=24$ for D and E; $n=3$ for F). Student's $t$ test, ${ }^{*} p<0.05,{ }^{* *} p<0.01$ and ${ }^{* * *} p<0.001$.

\subsection{A Genetic Screen of atm Mutant Suppressors}

The above data demonstrate that ATM functions as a negative regulator of leaf senescence. However, how ATM regulates leaf senescence is still unknown. To identify the components involved in ATM-mediated plant longevity, we performed forward genetic screens to identify the suppressors of atm mutant. Loss of ATM function causes multiple phenotypes, including early flowering, early senescence, decreased fertility, and increased plant height (Supplementary Figure S2A). Given that numerous environmental stresses such as shade or drought accelerate leaf senescence [4], while the fertility phenotype is relatively stable and easy to observe, we sought to identify the suppressors with restored fertility but not restored senescence phenotypes, termed as suppressor of atm in fertility (satmf). We assumed that the premature leaf senescence phenotypes of atm were suppressed along with the restoration of fertility. To this end, we carried out chemical mutagenesis by treating the seeds of wild-type Arabidopsis Col-0 and atm mutant with EMS [24]. Given that atm mutant is more sensitive to 
DNA-damage reagents such as EMS than wild-type Col-0 [20], seeds of Col-0 and atm-2 were treated with serial concentrations of EMS $(0.2 \%$ for $15 \mathrm{~h}, 0.3 \%$ for $12 \mathrm{~h}$, and $0.4 \% 10 \mathrm{~h})$ to seek for the optimal mutagenesis conditions (Supplementary Figure S2B). We found that mutagenesis with 0.3\% EMS for $12 \mathrm{~h}$ was suitable for atm seeds, which slightly affected seed germination (Supplementary Figure S2C), but produced various traits.

The model plant Arabidopsis has been widely used for forward genetic screening due to its short lifecycle, relatively little genome and abundant genetic resources [25-27]. In Arabidopsis, the genetic screening of mutants is first to mutate seeds through chemical (EMS), physical (fast neutron) or T-DNA insertional (activation tagging) mutagenesis, and then identify the mutants with expected phenotypes in M1/T1 or M2/T2 generation [28]. Plants with dominant mutation display phenotypes in M1 generation, and recessive mutations in M2 generation. However, most of the mutations cause no evident phenotypic changes due to genetic redundancy or inappropriate screening conditions [9]. Here, we found three fertility restored lines in the M1 generation, named satmf1, satmf2 and satmf3 (Figure 2A and Supplementary Figure S3A), suggesting that these mutants are caused by dominant mutations. To rule out the possibility of seed contamination, we firstly performed the genotyping analysis by using atm specific primers (Supplementary Table S1), and found that satmf1 3 are in atm mutant background (Supplementary Figure S3B). In order to further confirm their genetic backgrounds, the PCR products were mixed and used for sequencing, and we found, indeed, that satmf1 3 are in an atm background (Supplementary Figure S3C,D). Therefore, the homozygous mutants of satmf1 3 were used for the subsequent phenotypic analysis.
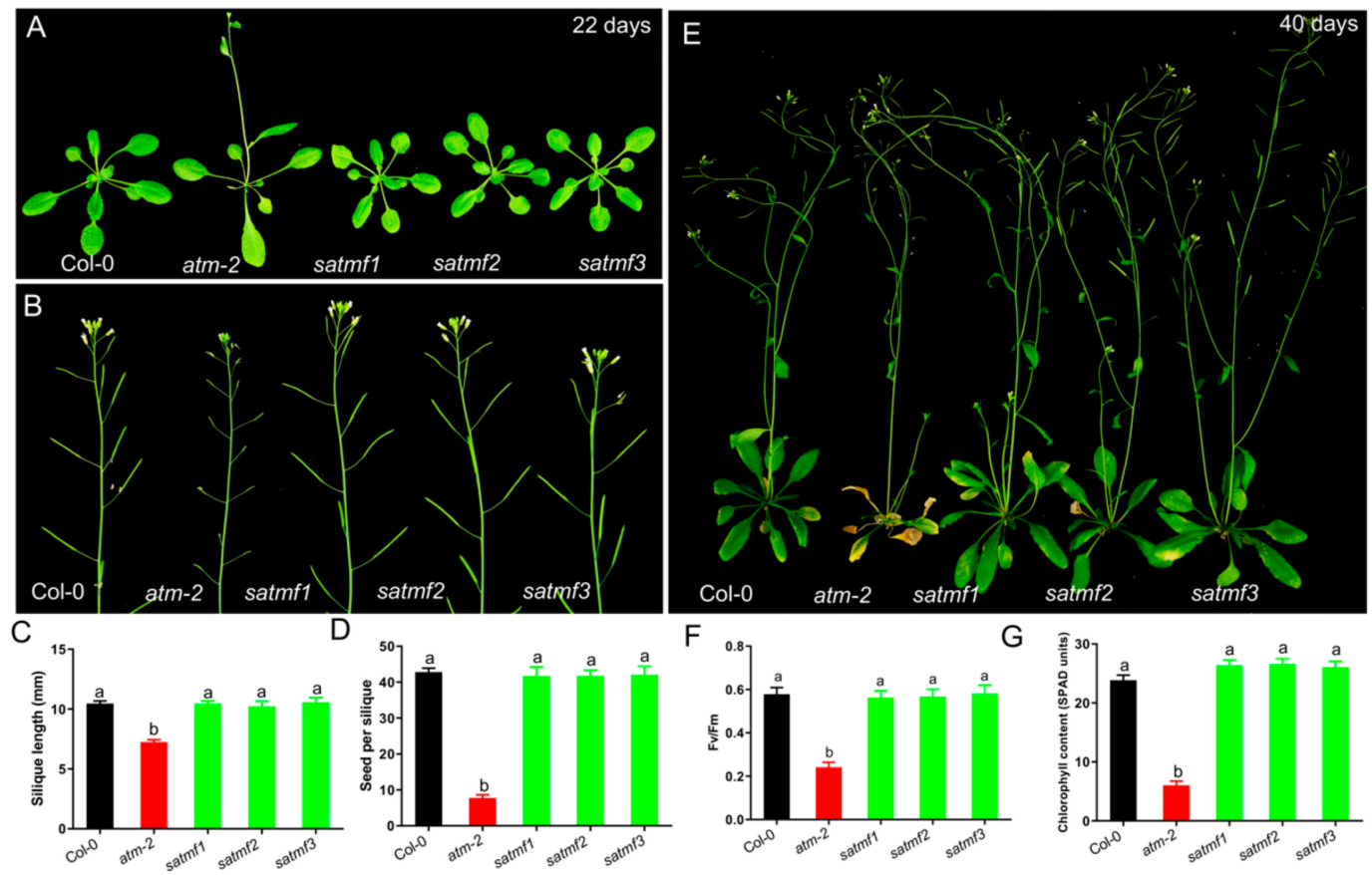

Figure 2. SATMF suppresses the early senescence phenotypes of atm mutant and improves its fertility. (A) Rosette leaves of 22-day-old plants of Col-0, atm-2, satmf1, satmf2 and satmf3. (B) Fertility of Col-0, atm-2, satmf1, satmf2 and satmf3 plants. Silique length (C), Number of seeds per silique (D), Senescence phenotypes (E) of 40-day-old plants of Col-0, atm-2, satmf1, satmf2 and satmf3. (F) Photochemical efficiency of PSII (Fv/Fm) and chlorophyll contents (G) in the third and fourth leaves of 35-day-old plants of indicated genetic backgrounds. Different letters represent significant differences (two-way ANOVA and post-hoc Tukey test, $p<0.05)$. 


\subsection{SATMF Suppresses the Early Senescence Phenotypes of atm Mutant and Improves Its Fertility}

To evaluate the effects of SATMF on restoring $\mathrm{atm}$ fertility and leaf senescence, we analyzed silique length, number of seeds per silique, the size of seeds, and rosette leaves of atm and satmf1 3. Gain-of-function of SATMF suppressed the early flowering phenotype of atm mutant (Figure 2A), and satmf1 3 plants had longer siliques (Col-0, $10.46 \pm 1.33 \mathrm{~mm}$; atm, $7.05 \pm 1.09 \mathrm{~mm}$; satmf1, $10.44 \pm 0.93 \mathrm{~mm}$; satmf2, $10.23 \pm 1.68 \mathrm{~mm}$; satmf3, $10.56 \pm 1.62 \mathrm{~mm}$ ) (Figure 2B,C), and more seeds per silique (Col-0, $42.68 \pm 6.32 ;$ atm, $7.83 \pm 4.8 ;$ satmf1, $41.46 \pm 9.13$; satmf2, $42.23 \pm 5.58 ;$ satmf3, $42.54 \pm 8.45)$ (Figure 2D), indicative of the restored fertility. As expected, the premature leaf senescence of the $\mathrm{atm}$ mutant was repressed in satmf1 3 (Figure 2E) with higher levels of PSII Photochemical efficiency $(\mathrm{Fv} / \mathrm{Fm})$ and chlorophyll contents (Figure 2F,G). The above data suggest that the gain of function of SATMF effectively restores the abnormal traits of atm mutant.

Although Arabidopsis is traditionally regarded as a key model organism for plant biology, it is increasingly clear that Arabidopsis is an important tool for us to understand the molecular mechanisms underlying human diseases [25,29]. A comparison of the annotated Arabidopsis and human genome sequences shows that there is also a high proportion of genes related to human diseases in Arabidopsis [25], including ATM (Supplementary Table S2). Although Arabidopsis and humans diverged 1.6 billion years ago, relatively recent studies have shown that protein functions and cellular processes are unexpectedly conserved between these distant species [30]. The use of Arabidopsis has elucidated many discoveries directly related to human health and disease [29]. In particular, Arabidopsis has been used to dissect cellular processes related to neurodegenerative diseases such as Alzheimer's disease, Parkinson's disease, and Friedrich ataxia [29]. The molecular cloning and functional analysis of SATMF will help to understand the potential regulatory mechanism of ATM in plants, and may also provide a reference for developing new treatments for the disease Ataxia-telangiectasia (A-T).

\subsection{Gain-of-Function of SATMF1 3 Restores the DSB Repair Efficiency in atm Background and Delays DSBs-Induced Leaf Senescence}

The above-reported data demonstrate dominant mutations of SATMF1 3 significantly improve the fertility compared with atm mutant, which prompted us to examine whether SATMF1 3 restored the DNA repair capacity in atm background. To this end, a comet assay was performed in 8-d-old third and fourth leaves of Col-0, atm and satmf1 3 plants before and after treatment with bleomycin (BLM) (Figure 3A), an inducer of DSBs [31]. A time-course analysis of DNA repair kinetics revealed that BLM treatment led to a sharp increase in DSBs that were efficiently repaired in 8-d-old leaves of Col-0 and satmf1-3 but not in atm mutant (Figure 3B), suggesting that DNA repair capacity for DSBs was efficiently restored by the gain of SATMF1 3 function. To confirm this observation, we detected the DSBs levels in 20-d-old leaves of Col-0 and satmf1 3 mutants. Accumulation of DSBs was evidently increased as leaves aged compared with 8-d-old leaves in Col-0 $(p<0.05)$. Higher levels of DSBs were found in 20-d-old leaves of atm in comparison with that in the leaves of Col-0 and satmf1 3 $(p<0.05)$, while no significant difference was observed between Col-0 and satmf1 3 mutant (Figure 3C), suggesting that SATMF1 3 efficiently suppress age-induced accumulation of damaged DNA.

To investigate the roles of SATMF1 3 in DSB-induced leaf senescence, the detached leaves of Col-0, atm and satmf1 3 were treated with $1 \mu \mathrm{g} / \mathrm{mL}$ BLM. The decline in the photochemical efficiency of PSII (Fv/Fm) upon treatment with BLM in atm leaves was more pronounced than that in Col-0 and satmf1 leaves (Figure 3D). A slightly but non-significantly higher level of Fv/Fm was observed in satmf1 compared to Col-0 at the same time points. These data suggest that SATMF1 plays an important role in suppressing DSB-induced leaf senescence. 
A
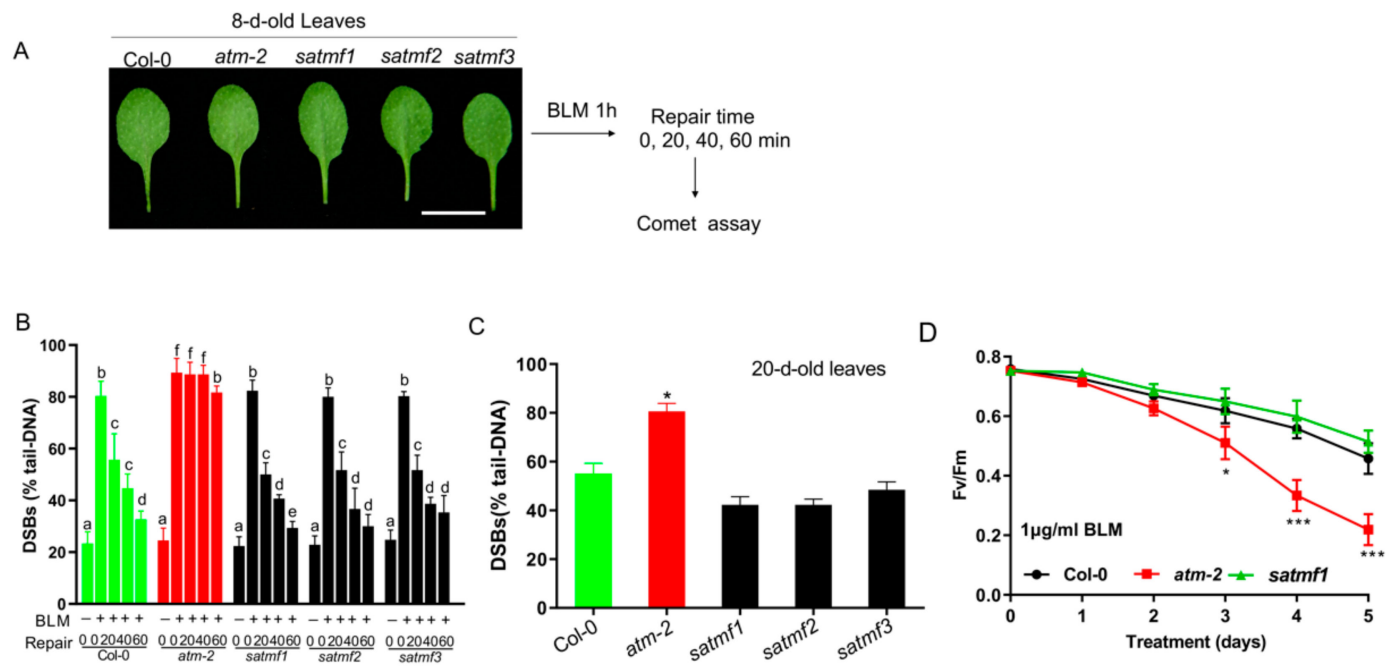

Figure 3. SATMF restores the DSB repair efficiency in atm-2 background and delays DSBs-induced leaf senescence. (A) Experimental schematic of evaluation of DNA repair ability in satmf1 3 by comet assay. The 8-d-old third and fourth leaves of Col-0, atm-2 and satmf1 3 upon treatment with $10 \mu \mathrm{g} / \mathrm{mL}$ BLM for $1 \mathrm{~h}$ were allowed to repair for $0,20,40$, or $60 \mathrm{~min}$, respectively, and used for comet assay. (B) Comet assay of the repair kinetics of the DSBs caused by BLM in the leaves in (A). Mean percentage of DNA in the tail at various recovery times after treatment was examined by comet assay. Three biological replicates were performed (mean \pm S.D.). Different letters represent significant differences (two-way ANOVA and post-hoc Tukey test, $p<0.05$ ). (C) Comet assay of DSBs levels in the third and fourth leaves of 20-d-old Col-0, atm-2 and satmf1 3 plants, evaluated as the mean percentage of DNA in the comet tail. Values shown represent the mean of three independent trials \pm S.D. Student's $t$-test, ${ }^{*} p<0.05$ (D) Photochemical efficiency of PSII (Fv/Fm) in the leaves of Col-0, atm-2, satmf1 plants upon treatment with $1 \mu \mathrm{g} / \mathrm{mL}$ BLM for up to $5 \mathrm{~d}$. Values shown represent the mean of three independent trials \pm S.D. Student's $t$-test: ${ }^{*} p<0.05 ;{ }^{* * *} p<0.001$.

\subsection{The ATM Loss-of-Function Mutant is Useful for Functional Genomics Research in Arabidopsis}

During the process of screening suppressors, we not only obtained the fertility-restored suppressors of atm mutant, but also found that the M1 plants derived from mutagenized atm seeds displayed more abundant phenotypes than that of Col-0. One of the mutants displayed longer petiole and hypocotyl than atm, reminiscent of Arabidopsis phytochrome (phy)-related mutants (Figure 4A,B). Sequencing analysis of genotyping PCR product revealed that a point mutation occurred in PHYA gene (Supplementary Figure S4), leading to a dominant negative (DN) phenotype (Figure 4A,B). We used these seeds to screen for ethylene-insensitive mutants and showed that more mutants were obtained in atm-2 background.

Given that ATM is an important component in DNA repair, DNA damage caused by alkylating agents such as EMS will cause more mutation in ATM loss of function mutant [32]. Damaged DNA can be repaired through multiple pathways, including direct repair by methylguanine methyl transferase (MGMT) and BER, or MMR (Figure 4C). If unrepaired, the methylguanine (MeG) preferably mispairs with T during DNA replication leading to transitions from G:C to A:T (Figure 4C) [32]. Phosphorylation of ATM activates MeG-dependent DNA damage response. MeG lesions induce sister chromatid exchanges (SCE) and chromosomal aberrations via MMR-dependent pathway in the second cell cycle [32]. The gaps and nicks are generated during this phase and can form DSB that are handled by ATM-mediated homologous repair. While loss of ATM function, cells become "methylation-tolerant" accumulating mutations and escape cell death in the presence of unrepaired $\mathrm{MeG}$, leading to generation of seeds with more mutated sites (Figure 4C). Collectively, our data suggest that atm mutant is a valuable tool for functional genomics research. 


$$
\text { A }
$$

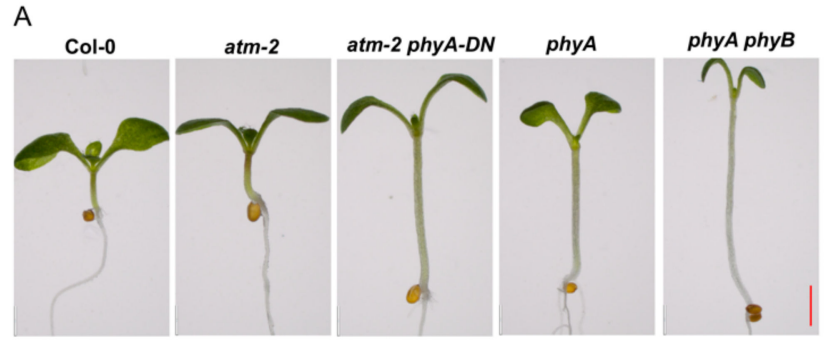

B

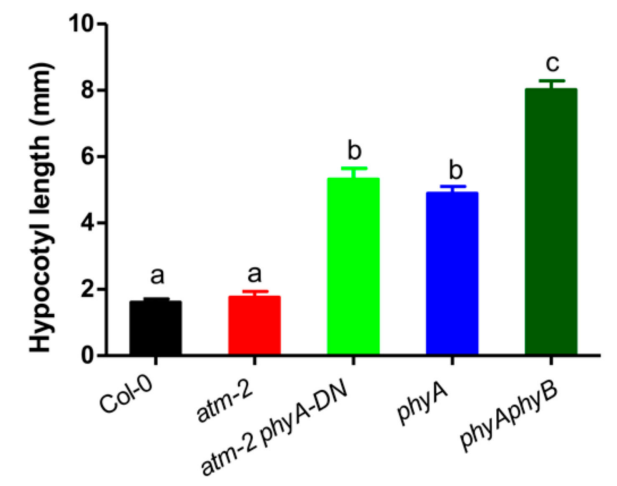

C

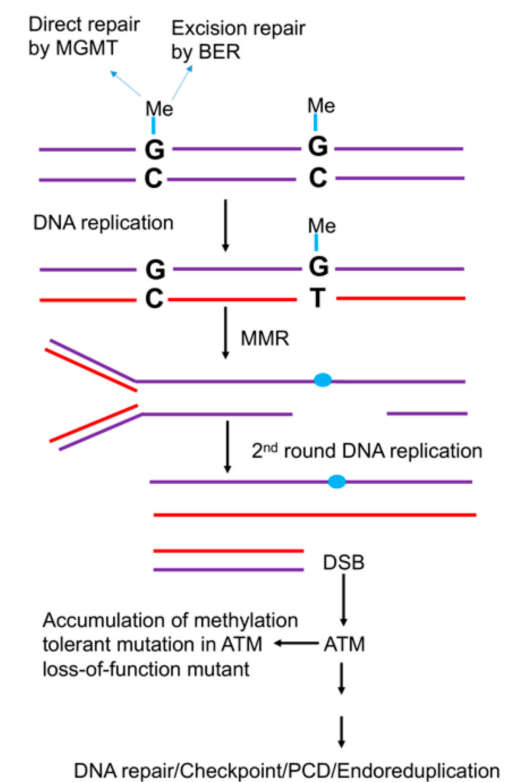

Figure 4. ATM loss-of-function mutants can be used to produce genetic materials in Arabidopsis. (A) Identification of a dominant negative (DN) mutant phyA-DN in EMS-treated atm mutant. After sowing, the petri dish with the seeds is placed at $4{ }^{\circ} \mathrm{C}$ for 3 days, and cultivated in a greenhouse at $22{ }^{\circ} \mathrm{C}$ for 5 days $(18 \mathrm{~L} / 6 \mathrm{D})$. (B) Measurement of hypocotyl length of the indicated seedlings. Different letters represent significant differences (two-way ANOVA and post-hoc Tukey test, $p<0.05$ ).

(C) Schematic representation of usage of $\mathrm{atm}$ for generating genetic materials.

\section{Materials and Methods}

\subsection{Plant Materials and Growth Conditions}

All the Arabidopsis plant materials used in this study were in the Col-0 background. Plants were grown in an environmentally controlled growth room under long-day conditions $(16 \mathrm{~L} / 8 \mathrm{D})$ at $22^{\circ} \mathrm{C}$ and $65 \%$ relative humidity.

\subsection{Plasmid Construction and Generation of Transgenic Plants}

To generate 35S:GFP-ATM/Col-0, the full-length ATM CDS sequence was amplified and then inserted into pEGAD vector [33]. To construct ProATM:GUS/Col-0, a $3 \mathrm{~kb}$ genomic promoter sequence was amplified and inserted into pBI101 vector [34]. The amplified fragments were inserted into respective vectors by using the in-fusion enzyme (TAKARA, Shiga, Japan). All constructs were transformed into Agrobacterium tumefaciens cells (strain GV3101) which was used to transform Col-0 plants by the floral dip method [35]. All primer sequences used here are listed in Supplementary Table S1.

\subsection{Assays of Age-Dependent and Bleomycin-Induced Leaf Senescence}

To assess age-dependent leaf senescence, third and fourth rosette leaves were harvested from 40-d-old plants. For bleomycin (BLM)-induced leaf senescence, 8-d-old third and fourth rosette leaves were detached and floated on $3 \mathrm{mM}$ MES buffer ( $\mathrm{pH}$ 5.7) supplemented with or without $1 \mu \mathrm{g} / \mathrm{mL}$ BLM for up to $5 \mathrm{~d}$ under long-day conditions. Photochemical efficiency of photosystem II (Fv/Fm) was measured using a MultispeQ (East Lansing, MI, USA) instrument, and chlorophyll contents were measured using a chlorophyll meter SPAD502 Plus (Konica Minolta, Hino-shi, Tokyo, Japan). 


\subsection{EMS Mutagenesis of Arabidopsis Seeds}

Ethyl methanesulfonate (EMS) mutagenesis of atm seeds was performed in the laboratory of Prof. Hong Gil Nam (DGIST, Korea) according to protocols as described previously [24]. Given that atm mutant is more sensitive to EMS than wild-type Col-0 (Supplementary Figure S2C), seeds of Col-0 and $\mathrm{atm}$ were treated with EMS with a final concentration of $0.2 \%$ (1.5 g, 75,000 seeds), $0.3 \%$ ( $2 \mathrm{~g}$, $\sim 100,000$ seeds) and $0.4 \%$ ( $1 \mathrm{~g}, \sim 50,000$ seeds) for $15 \mathrm{~h}, 12 \mathrm{~h}$ and $10 \mathrm{~h}$ respectively. M1 seeds were sown in soil uniformly with the use of a squeeze bottle and used for direct screening for dominant mutants, and M2 seeds were subjected to screen for recessive mutants.

\subsection{Comet Assay of DSBs}

DSB levels were determined using a comet assay with a neutral protocol, measuring the amount of DNA in the tail, as described previously [36]. Images of comets were captured at 20-fold magnification using a monochrome CCD camera. Images were analyzed with the Image J software plugin OpenComt (http://www.cometbio.org/) [37]. Each experimental point is represented by the mean value from three replicates of electrophoresis gels.

\subsection{RNA Isolation and Real-Time PCR}

Total RNA was isolated using plant RNA kits (ER301; TransGen Biotech, Beijing, China), and complementary DNA was produced with cDNA Synthesis kit (AT341; TransGen Biotech) according to the manufacturers' protocols. Transcript levels were assessed by qPCR kit (AQ111; TransGen Biotech) using Applied Biosystems 7500 Real-Time PCR System (Thermo Scientific, Waltham, MA, USA). The primers used in this study are listed in Supplementary Table S1.

\section{Conclusions}

Previous results demonstrated that ATM functions as a negative regulator of plant aging through controlling age-induced DSBs accumulation, which has significant implications for the conservation of aging mechanisms in animals and plants [12]. We obtained three suppressors of atm mutant with delayed senescence and restored fertility. Functional analysis of SATMF will help to understand the underlying regulatory mechanism of ATM in plants, and may also provide a reference for developing new treatments for the disease Ataxia-telangiectasia (A-T). A-T is a neurodegenerative disease [38], and no curative medication is found to effectively treat this disease [39]. Although Arabidopsis is traditionally viewed as the key model organism for plant biology, it is becoming gradually clear that Arabidopsis represents an invaluable tool for understanding molecular mechanisms underlying human diseases [29]. Additionally, our data also show that atm mutant might be a valuable tool for functional genomics research in Arabidopsis.

Supplementary Materials: Supplementary materials can be found at http://www.mdpi.com/1422-0067/21/21/ 8120/s1.

Author Contributions: Conceptualization, Z.L.; methodology, Z.L., Y.Z. and H.-L.W.; investigation, Y.Z. and H.-L.W.; writing—original draft preparation, Z.L. and Y.Z.; writing—review and editing, Z.L., Y.Z. and Y.G.; supervision, H.G. All authors have read and agreed to the published version of the manuscript.

Funding: This work was supported by the National Natural Science Foundation of China (31970196 to Z.L.; 31570286 to H.G.; 31900173 to H.-L.W.), Chinese Postdoctoral Science Foundation (2019M650514 to Y.Z.; 2019M650516 to H.-L.W.; 2020M670544 to Y.G.), Shenzhen Science and Technology Program (No. KQTD20190929173906742 to H.G.) and the National Key Research and Development Program of China (No. 2019YFA0903904 to H.G.), and the startup funding for plant aging research from Beijing Advanced Innovation Center for Tree Breeding by Molecular Design, Beijing Forestry University.

Acknowledgments: We thank Hong Gil Nam (Center for Plant Aging Research, IBS, Korea) and Hye Ryun Woo (DGIST, Korea) for valuable advice and discussions.

Conflicts of Interest: The authors declare no conflict of interest. 


\section{Abbreviations}

$\begin{array}{ll}\text { ATM } & \text { Ataxia Telangiectasia Mutated } \\ \text { ATR } & \text { ATM- and RAD3-related } \\ \text { DSB } & \text { Double-strand Break } \\ \text { SATMF } & \text { Suppressor of } a \text { atm in Fertility } \\ \text { BER } & \text { Base Excision Repair } \\ \text { HR } & \text { Homologous Recombination } \\ \text { NHEJ } & \text { Non-Homologous End Joining } \\ \text { EMS } & \text { Ethyl MethaneSulfonate }\end{array}$

\section{References}

1. Guo, Y.; Gan, S.S. Translational researches on leaf senescence for enhancing plant productivity and quality. J. Exp. Bot. 2014, 65, 3901-3913. [CrossRef]

2. Woo, H.R.; Kim, H.J.; Nam, H.G.; Lim, P.O. Plant leaf senescence and death-regulation by multiple layers of control and implications for aging in general. J. Cell Sci. 2013, 126, 4823-4833. [CrossRef]

3. Lim, P.O.; Kim, H.J.; Nam, H.G. Leaf senescence. Annu. Rev. Plant Biol. 2007, 58, 115-136. [CrossRef]

4. Guo, Y.; Gan, S. Leaf senescence: Signals, execution, and regulation. Curr. Top. Dev. Biol. 2005, 71, 83-112. [PubMed]

5. Oh, S.A.; Park, J.H.; Lee, G.I.; Paek, K.H.; Park, S.K.; Nam, H.G. Identification of three genetic loci controlling leaf senescence in Arabidopsis thaliana. Plant J. 1997, 12, 527-535. [CrossRef] [PubMed]

6. Nam, H.G. The molecular genetic analysis of leaf senescence. Curr. Opin. Biotechnol. 1997, 8, $200-207$. [CrossRef]

7. Lim, P.O.; Woo, H.R.; Nam, H.G. Molecular genetics of leaf senescence in Arabidopsis. Trends Plant Sci. 2003, 8, 272-278. [CrossRef]

8. Guo, P.; Li, Z.; Huang, P.; Li, B.; Fang, S.; Chu, J.; Guo, H. A tripartite amplification loop involving the transcription factor WRKY75, salicylic acid, and reactive oxygen species accelerates leaf senescence. Plant Cell 2017, 29, 2854-2870. [CrossRef] [PubMed]

9. Li, Z.; Woo, H.R.; Guo, H. Genetic redundancy of senescence-associated transcription factors in Arabidopsis. J. Exp. Bot. 2018, 69, 811-823. [CrossRef]

10. Woo, H.R.; Kim, H.J.; Lim, P.O.; Nam, H.G. Leaf senescence: Systems and dynamics aspects. Annu. Rev. Plant Biol. 2019, 70, 347-376. [CrossRef]

11. White, R.R.; Vijg, J. Do DNA double-strand breaks drive aging? Mol. Cell 2016, 63, 729-738. [CrossRef] [PubMed]

12. Li, Z.; Kim, J.H.; Kim, J.; Lyu, J.I.; Zhang, Y.; Guo, H.; Nam, H.G.; Woo, H.R. ATM suppresses leaf senescence triggered by DNA double-strand break through epigenetic control of senescence-associated genes in Arabidopsis. New Phytol. 2020, 227, 473-484. [CrossRef] [PubMed]

13. Gorbunova, V.; Seluanov, A.; Mao, Z.; Hine, C. Changes in DNA repair during aging. Nucleic Acids Res. 2007, 35, 7466-7474. [CrossRef] [PubMed]

14. Opresko, P.L.; Cheng, W.H.; von Kobbe, C.; Harrigan, J.A.; Bohr, V.A. Werner syndrome and the function of the Werner protein; what they can teach us about the molecular aging process. Carcinogenesis 2003, 24, 791-802. [CrossRef]

15. Goldman, R.D.; Shumaker, D.K.; Erdos, M.R.; Eriksson, M.; Goldman, A.E.; Gordon, L.B.; Gruenbaum, Y.; Khuon, S.; Mendez, M.; Varga, R.; et al. Accumulation of mutant lamin A causes progressive changes in nuclear architecture in Hutchinson-Gilford progeria syndrome. Proc. Natl. Acad. Sci. USA 2004, 101, 8963-8968. [CrossRef]

16. Shiloh, Y.; Ziv, Y. The ATM protein kinase: Regulating the cellular response to genotoxic stress, and more. Nat. Rev. Mol. Cell Biol. 2013, 14, 197-210. [CrossRef]

17. Savitsky, K.; Bar-Shira, A.; Gilad, S.; Rotman, G.; Ziv, Y.; Vanagaite, L.; Tagle, D.A.; Smith, S.; Uziel, T.; Sfez, S.; et al. A single ataxia telangiectasia gene with a product similar to PI-3 kinase. Science 1995, 268, 1749-1753. [CrossRef] [PubMed]

18. Kang, H.T.; Park, J.T.; Choi, K.; Kim, Y.; Choi HJ, C.; Jung, C.W.; Lee, Y.S.; Park, S.C. Chemical screening identifies ATM as a target for alleviating senescence. Nat. Chem. Biol. 2017, 13, 616-623. [CrossRef] [PubMed] 
19. Unsal-Kacmaz, K.; Sancar, A. Quaternary structure of ATR and effects of ATRIP and replication protein A on its DNA binding and kinase activities. Mol. Cell Biol. 2004, 24, 1292-1300. [CrossRef]

20. Culligan, K.M.; Robertson, C.E.; Foreman, J.; Doerner, P.; Britt, A.B. ATR and ATM play both distinct and additive roles in response to ionizing radiation. Plant J. 2006, 48, 947-961. [CrossRef]

21. Britt, A.B. DNA Damage and Repair in Plants. Annu. Rev. Plant Physiol Plant Mol. Biol. 1996, 47, 75-100. [CrossRef] [PubMed]

22. Garcia, V.; Bruchet, H.; Camescasse, D.; Granier, F.; Bouchez, D.; Tissier, A. AtATM is essential for meiosis and the somatic response to DNA damage in plants. Plant Cell 2003, 15, 119-132. [CrossRef]

23. White, R.R.; Milholland, B.; de Bruin, A.; Curran, S.; Laberge, R.M.; van Steeg, H.; Campisi, J.; Maslov, A.Y.; Vijg, J. Controlled induction of DNA double-strand breaks in the mouse liver induces features of tissue ageing. Nat. Commun. 2015, 6, 6790. [CrossRef] [PubMed]

24. Kim, Y.; Schumaker, K.S.; Zhu, J.K. EMS mutagenesis of Arabidopsis. Methods Mol. Biol. 2006, 323, 101-103.

25. Arabidopsis Genome, I. Analysis of the genome sequence of the flowering plant Arabidopsis thaliana. Nature 2000, 408, 796-815. [CrossRef] [PubMed]

26. Berardini, T.Z.; Reiser, L.; Li, D.; Mezheritsky, Y.; Muller, R.; Strait, E.; Huala, E. The Arabidopsis information resource: Making and mining the "gold standard" annotated reference plant genome. Genesis 2015, 53, 474-485. [CrossRef] [PubMed]

27. Clouse, S.D.; Langford, M.; McMorris, T.C. A brassinosteroid-insensitive mutant in Arabidopsis thaliana exhibits multiple defects in growth and development. Plant Physiol. 1996, 111, 671-678. [CrossRef] [PubMed]

28. Javorka, P.; Raxwal, V.K.; Najvarek, J.; Riha, K. artMAP: A user-friendly tool for mapping ethyl methanesulfonate-induced mutations in Arabidopsis. Plant Direct 2019, 3, e00146. [CrossRef]

29. Xu, X.M.; Moller, S.G. The value of Arabidopsis research in understanding human disease states. Curr. Opin. Biotechnol. 2011, 22, 300-307. [CrossRef]

30. Rubin, G.M.; Yandell, M.D.; Wortman, J.R.; Gabor Miklos, G.L.; Nelson, C.R.; Hariharan, I.K.; Fortini, M.E.; Li, P.W.; Apweiler, R.; Fleischmann, W.; et al. Comparative genomics of the eukaryotes. Science 2000, 287, 2204-2215. [CrossRef]

31. Steighner, R.J.; Povirk, L.F. Bleomycin-induced DNA lesions at mutational hot spots: Implications for the mechanism of double-strand cleavage. Proc. Natl. Acad. Sci. USA 1990, 87, 8350-8354. [CrossRef] [PubMed]

32. Klapacz, J.; Pottenger, L.H.; Engelward, B.P.; Heinen, C.D.; Johnson, G.E.; Clewell, R.A.; Carmichael, P.L.; Adeleye, Y.; Andersen, M.E. Contributions of DNA repair and damage response pathways to the non-linear genotoxic responses of alkylating agents. Mutat. Res. Rev. Mutat. Res. 2016, 767, 77-91. [CrossRef] [PubMed]

33. Cutler, S.R.; Ehrhardt, D.W.; Griffitts, J.S.; Somerville, C.R. Random GFP: cDNA fusions enable visualization of subcellular structures in cells of Arabidopsis at a high frequency. Proc. Natl. Acad. Sci. USA 2000, 97, 3718-3723. [CrossRef] [PubMed]

34. Jefferson, R.A.; Kavanagh, T.A.; Bevan, M.W. GUS fusions: Beta-glucuronidase as a sensitive and versatile gene fusion marker in higher plants. Embo J. 1987, 6, 3901-3907. [CrossRef]

35. Clough, S.J.; Bent, A.F. Floral dip: A simplified method for Agrobacterium-mediated transformation of Arabidopsis thaliana. Plant J. 1998, 16, 735-743. [CrossRef]

36. Menke, M.; Angelis, K.J.; Schubert, I. Detection of specific DNA lesions by a combination of comet assay and FISH in plants. Environ. Mol. Mutagen. 2000, 35, 132-138. [CrossRef]

37. Gyori, B.M.; Venkatachalam, G.; Thiagarajan, P.S.; Hsu, D.; Clement, M.V. OpenComet: An automated tool for comet assay image analysis. Redox Biol. 2014, 2, 457-465. [CrossRef]

38. Boder, E. Ataxia-telangiectasia: An overview. Kroc Found. Ser. 1985, 19, 1-63.

39. Ilg, W.; Bastian, A.J.; Boesch, S.; Burciu, R.G.; Celnik, P.; Claassen, J.; Feil, K.; Kalla, R.; Miyai, I.; Nachbauer, W.; et al. Consensus paper: Management of degenerative cerebellar disorders. Cerebellum 2014, 13, 248-268. [CrossRef]

Publisher's Note: MDPI stays neutral with regard to jurisdictional claims in published maps and institutional affiliations. 